\section{¿Presión o legitimación? Poder y alternativas en el diseño y adopción de la reforma de salud en Costa Rica, 1988-1998}

Pressure or legitimization? Power and alternatives in the planning and adoption of health reforms in Costa Rica, 1988-1998

Juliana Martínez Franzoni Instituto de Investigaciones Sociales Universidad de Costa Rica Ciudad Universitaria Rodrigo Facio

Apartado Postal 49-2060 - San

Pedro de Montes de Oca julianam@cariari.ucr.ac.cr
MARTÍNEZ FRANZONI, J.: ¿Presión o legitimación? Poder y alternativas en el diseño y adopción de la reforma de salud en Costa Rica, 1988-1998.

História, Ciências, Saúde - Manguinhos, v. 13, n. 3, p. 591-622, julio-sept. 2006.

La formación de políticas responde a lógicas de presión y legitimación que se combinan de distintas maneras. Este trabajo analiza el diseño y adopción de dos políticas de reforma de salud realizadas en Costa Rica entre 1988 y 1998. El trabajo se pregunta si son los partidos políticos, las instituciones financieras internacionales y las elites tecnoburocráticas sectoriales factores explicativos suficientes. La evidencia empírica sugiere que además de intereses particulares de los actores involucrados, es necesario considerar las agendas de reforma sectorial internacionalmente disponibles. El trabajo aproxima este debate a la formación de políticas en América Latina y facilita los análisis que permiten tender puentes entre otras etapas de la formación de políticas y otros momentos históricos de la construcción del Estado.

PALABRAS-CLAVE: políticas públicas; Estado; salud; reformas.

MARTÍNEZ FRANZONI, J.: Pressure or legitimization? Power and alternatives in the planning and adoption of health reforms in Costa Rica, 1988-1998.

História, Ciências, Saúde - Manguinhos, v. 13, n. 3, p. 591-622, July-Sept. 2006.

Policies are made in response to the rationale of pressure and legitimization, which join forces in many different ways. This work analyzes the planning and adoption of the health reforms undertaken in Costa Rica between 1988 and 1998. It questions whether political parties, international financial institutions and the technical and bureaucratic elites in each sector can be taken as sufficiently explanatory of themselves. Empirical evidence would suggest that apart from investigating the individual interests of the agents involved, one must also consider the sector reforms that are actually available internationally. The paper draws this discussion into the larger scenario of policy making in Latin America and draws links between this and other stages in policy making and other moments in the construction of the State.

KEYWORDS: public policy; State; health; reforms. 
Politics finds its sources not only in power but also in uncertainty men collectively wondering what to do... Governments not only 'power'... they also puzzle... Much political interaction has constituted a process of social learning expressed through policy.

Heclo, in Hall, 1993

In the modern period it has been elites within nation-states who have been particularly concerned with cross-societal emulation and selective incorporation, and who have indeed made such activities a crucial aspect of nation-state formation and transformation.

Robertson, 1994, p. 113

\begin{abstract}
A mérica Latina atraviesa profundas transformaciones en sus políticas sociales. El estudio de políticas pasadas y contemporáneas puede contribuir a una mayor comprensión e identificación de recomendaciones para las políticas futuras. Requiere, sin embargo, profundizar el diálogo y la colaboración entre la investigación histórica, con fortalezas en la caracterización de procesos en distintas etapas de la formación de los Estados nacionales en la región, y la investigación sociológica y politológica, con fortalezas en la exploración de marcos interpretativos replicables y superables. Se trata, desde mi punto de vista, de insertar el análisis empírico en marcos interpretativos compartibles.

Desde la sociología y la ciencia política, las investigaciones latinoamericanas en materia de política social contemporánea enfatizan las características de las políticas, sea en términos de principios (equidad, solidaridad, subsidiaridad), de instrumentos (de regulación, financiamiento, prestación) o de servicios (usualmente específicos a cada sector de política social como salud, educación, pensiones) (Mesa-Lago, 1994; Filgueira, 1998). Está menos generalizado el estudio de determinantes o factores explicativos que vuelve la mirada a la formación de políticas en los distintos momentos del ciclo, desde la definición de problemas y diseño de soluciones, hasta su adopción e implementación (Jones, 1970; Anderson, 1975). ${ }^{1}$ ¿Son las reformas de política principalmente producto de la presión (de grupos económicos, de organismos internacionales) o de la búsqueda de aceptación? (acordes a los avances del conocimiento científico, de las recomendaciones de organismos internacionales, de la modernización). En nuestra región, las perspectivas teóricas han privilegiado el análisis de las relaciones de poder en detrimento del peso relativo de los paradigmas e ideas disponibles para la elaboración de dichos intereses. Esto ha ocurrido tanto en estudios centrados en el nivel nacional (por
\end{abstract}


ejemplo, Carmelo Mesa-Lago, 1978, 1989; Fernando Filgueira, 1998) como en el papel de los actores internacionales (por ejemplo, Lynn Morgan, 1993). Excepción a la regla es el trabajo de Steven Palmer (1998) que, enfocado en el papel de la Fundación Rockefeller en Costa Rica, cuestiona que ésta realmente supiera lo que venía a hacer al Tercer Mundo (como da por sentado Morgan) y da mayor lugar a la construcción de agendas de políticas públicas que tienen lugar en nuestra región del mundo (Martínez Franzoni, 2000). ${ }^{2}$

A partir del clásico trabajo de Lowi (1964) parece ganar terreno la idea de que la lógica de formación de políticas depende fuertemente del tipo de políticas (Thun, 2003; Weyland, 2005). Según Lowi, esta lógica varía según sean estas regulatorias (relacionadas con 'reglas de juego'), distributivas (relativas a la asignación de recursos a clientelas específicas sin que medie consideración de limitación de recursos) o redistributivas (relacionadas con la transferencia de recursos de unos a otros sectores o grupos de la sociedad). Siendo las políticas sociales políticas redistributivas, Kurt Weyland argumenta que los cambios están dominados por la presión. El análisis de las dos rutas reformistas de su sistema único de salud experimentado por Costa Rica entre 1988 y 1998, acota el argumento de Weyland y sugiere que siendo plausible para la etapa de implementación de políticas, no lo es para los momentos de formulación y adopción. ${ }^{3}$ Un examen inductivo de los distintos factores explicativos en juego permite alumbrar el peso relativo que las ideas, en particular la disponibilidad de un 'menú' internacional de políticas públicas, tuvo en los momentos de formulación y adopción de una y otra ruta reformista. ${ }^{4}$

\section{La reforma de salud en Costa Rica}

Entre 1988 y 1998, dos intentos de reforma del sistema de salud costarricense respondieron a generaciones u olas de transformación institucional mundiales, orientadas a mejorar el rendimiento del financiamiento público de los servicios de salud. El primer intento promovió medidas de privatización y descentralización, mientras que el segundo introdujo mecanismos de competencia entre servicios públicos. En el primer caso, las reformas impulsaron contratos con proveedores privados. Entre 1988 y 1991, se crearon tres clínicas y otras tantas se sometieron a consideración de las autoridades del seguro social. Frente a estas medidas, el veto principal provino de los cuadros tecnoburocráticos del seguro social, el cual restringió fuertemente la expansión de la contratación de servicios privados y vetó su extensión a más usuarios y niveles del sistema de salud.

El segundo intento reformista (1994-1998) principalmente promovió una reorganización gerencial del sector a través de competencia regulada entre dependencias públicas. Específicamente, 
la reforma estableció compromisos de gestión entre servicios públicos y las oficinas administrativas centrales del seguro social. Hacia fines de 1998, esta política había logrado avanzar y la oposición, aunque existente (por ejemplo, proveniente de sindicatos de trabajadores y asociaciones de médicos), estaba fragmentada y lejos de tener capacidad de bloqueo. Al revés del primer intento reformista que ponía en cuestión la conveniencia de la provisión directa de servicios por parte del estado, el segundo la expandió y consolidó. Paradójicamente, el primer intento fue promovido bajo una administración de gobierno de tradición socialdemócrata (Partido de Liberación Nacional, 1986-1990) y el segundo por una administración conservadora (Partido de Unidad Social-Cristiana, 1990-1994).

A partir de entrevistas con autoridades políticas y técnicas del seguro social realizadas entre 1994 y 1996, este trabajo examina el efecto de la alternancia político partidaria en la administración nacional del país (de una de corte socialdemócrata a una de corte neoliberal); los acuerdos firmados con las instituciones financieras internacionales (entre un acuerdo macro-económico y uno sectorial, respectivamente); y los cambios en el papel negociador de los cuadros tecnoburocráticos del seguro social (de un papel marginal a otro de activa participación). Del análisis se desprende que, considerados individualmente, el accionar de estos actores ha sido complementario y necesario pero a la vez insuficiente para explicar el diseño y adopción de ambas reformas. Finalmente, es preciso tener en cuenta las alternativas reformistas disponibles en uno y otro momento y su adecuación a la matriz política e institucional del sector salud costarricense. Mientras en un primer momento dicha alternativa estaba fuertemente basada en la privatización y la descentralización de los servicios de salud, en un segundo momento las alternativas disponibles promovían además la adopción de arreglos organizacionales provenientes del sector privado dentro del propio sector público.

Los trabajos sobre la reforma del estado en América Latina, en particular aquellos relativos a las reformas institucionales en el marco del ajuste estructural de las economías, han tendido a enfatizar el papel de los actores y su correspondiente movilización de intereses frente a cada reforma, sean dichos actores sectoriales, nacionales o internacionales. En este marco, suele considerarse que las políticas fracasan o son exitosas de acuerdo con la capacidad que los actores tengan de generar apoyos y activar vetos, dándose pues por sentado que dichos actores cuentan con agendas que claramente establecen cuál es el diseño institucional más adecuado para la protección de sus intereses. Más aún, cuando la literatura sí examina la disponibilidad de agendas o modelos reformistas, éstos suelen considerarse como resultantes de la negociación entre actores 
antes que como insumos para la propia determinación de las posiciones que asumen los actores en la negociación de políticas que les afectan. ${ }^{5}$ El papel de las ideas y su capacidad explicativa de las reformas actuales ha sido examinado como parte del campo de política comparada y relaciones internacionales (Jacobsen, 1995). Esencialmente, de este debate se desprende la necesidad de articular las relaciones de poder e interés con el conocimiento, las agendas y los modelos disponibles en función de las cuales los actores pueden legítimamente defender sus intereses. Hasta el momento, sin embargo, los estudios de las reformas latinoamericanas en general han ignorado este debate. Alejándome de las explicaciones dominantes respecto a la formación de políticas en este trabajo, argumento la importancia de combinar éstas con la disponibilidad de ideas y modelos para la acción.

Dado que estos ejes reformistas no son producidos domésticamente por parte de ningún país en particular, necesitan de alguna manera entrar en la arena doméstica, relacionarse con legados político-institucionales existentes, y tener contacto con aquellos actores que los pueden llevar a cabo. Por eso, finalmente examino los mecanismos que permiten dicho acceso, prestándole particular atención al papel de las elites domésticas en la búsqueda, adaptación y adopción de opciones disponibles internacionalmente.

\section{Las políticas reformistas como resultante de las preferencias político partidarias}

¿Hay una relación directa entre las preferencias declaradas de los partidos políticos costarricenses y las reformas de la salud que éstos impulsaron estando en el gobierno? ¿En qué medida la alternancia partidaria tuvo un efecto sobre las políticas reformistas promovidas entre 1988 y 1998? Se trata pues de establecer si, y en qué grado, los partidos "hicieron una diferencia" (Rose, 1984) en la aparición, contenidos y resultados de cada uno de los intentos reformistas. Más concretamente, la hipótesis a examinar es en qué medida las reformas de salud costarricenses se correspondieron con las preferencias ideológicas de los partidos políticos bajo cuyas administraciones de gobierno se iniciaron.

Entre 1988 y 1998, dos partidos dominaron la escena política costarricense: el Partido de Liberación Nacional o PLN (1986-1990; 1994-1998) y el Partido Unidad Social-Cristiana o PUSC (1990-1994; 1998-2002). ${ }^{6}$ Ambos partidos estuvieron al frente de administraciones que ganaron con muy escaso margen electoral, lo cual promovió sucesivos procesos de consulta y negociación entre los partidos. ${ }^{7}$ Si analizamos las preferencias ideológicas históricas del PLN y del PUSC, éstas no nos dicen demasiado acerca de las políticas llevadas a cabo durante los años 80 y 90, cuando Costa Rica llevó adelante 
el ajuste macro-económico. Ambos partidos tuvieron un papel importante en este sentido, aunque en mayor medida el PLN que el PUSC dado que estuvo en el gobierno durante la mayor parte de la década de los 80, cuando dicho ajuste tuvo lugar. ${ }^{8}$ A cambio de estas políticas, los gobiernos del PLN lograron la 'luz verde' de las instituciones financieras internacionales - IFIs que les habían retirado completamente el apoyo financiero al país a partir de la moratoria de la deuda externa declarada durante la administración de Carazo (1978-1982).

En lo que respecta a las políticas sociales, y con excepción del sector salud, no hubo transformaciones institucionales (aunque sí programáticas) significativas de las políticas sociales ni intentos en esa dirección. ${ }^{9}$ Entre 1988 y 1992, en el sector salud se llevó adelante un primer intento reformista, orientado a desarrollar nuevas modalidades de provisión de servicios. La iniciativa fue de las autoridades sectoriales, a partir de propuestas técnicas provenientes de médicos de la institución. Médico también, el presidente ejecutivo, Dr. Guido Miranda, había sido nombrado en el cargo durante la administración de Monge y exitosamente sacado a la Caja - Caja Costarricense del Seguro Social - de la profunda crisis financiera en la que ésta se encontraba. Una vez alcanzada la estabilidad financiera, Miranda promovió transformaciones institucionales, principalmente orientadas a aumentar la relación costo-efectividad de los servicios del programa de salud del seguro social. A pesar de que fueron varios los programas piloto destinados a innovar en los servicios de atención y administración, el más ambicioso en su implementación y proyección, como posible ruta reformista del sistema en su conjunto, fue el de creación y contratación de cooperativas médicas, originalmente a través de la Cooperativa de Salud Coopersalud, que prestaría servicios en la comunidad de Pavas. ${ }^{10}$

Dentro del PLN no había consenso respecto a la conveniencia de la contratación de terceros, ni entre sus propios cuadros técnicos dentro del seguro social ni fuera de éste. Estos desacuerdos se reflejaron, por ejemplo, en la oposición de cuadros tecnoburocráticos del seguro social, simpatizantes del PLN. Fuera del seguro social, dicha oposición se manifestó en la postura de la Contraloría General de la Nación, agencia autónoma contralor de las instituciones públicas, la cual consideró la iniciativa inconstitucional. El levantamiento del veto de la Contraloría requirió que las autoridades de la Caja reinterpretaran uno de los artículos de la Ley Constitutiva, el cual faculta a la Caja para poner en práctica 'programas especiales' destinados a la mejora del servicio, siempre y cuando éstos mantuvieran los principios de universalidad, equidad y solidaridad, pilares del sistema. La aprobación de la iniciativa solo se logró luego de negociaciones entre la Caja, el Presidente de la República y la Contraloría, y en el contexto de presiones públicas y opiniones 
divididas dentro del PLN, tanto a favor como en contra de la contratación externa de servicios.

En 1990, cuando el PUSC accedió al gobierno (Calderón, 19901994), las evaluaciones llevadas a cabo por consultores externos en relación a la efectividad y calidad de los servicios de salud mediante la contratación externa de servicios eran muy positivas (por ejemplo, Usaid) (Fiedler \& Rigoli, 1991). Más aún, la satisfacción de los usuarios, individual y colectiva, medida a través de encuestas y de las organizaciones comunitarias, respectivamente, era muy alta. La aparente afinidad ideológica del PUSC con medidas de privatización y de descentralización del estado anunciadas durante la campaña electoral, combinadas con el apoyo popular e internacional a dichas medidas, hizo esperar que la administración profundizara dichas medidas mediante una expansión de la contratación externa de servicios (Trejos \& Güendell, 1994). De hecho, y a pesar de una gran cautela pública, durante el comienzo de la administración del PUSC se crearon otras dos cooperativas, alcanzando a unas 150.000 personas e iniciándose conversaciones con grupos médicos interesados en administrar clínicas fuera del área metropolitana de San José. ${ }^{11}$ En los hechos, al menos en el sector social, no se llevaron a cabo transformaciones institucionales importantes, si bien sí existieron reducciones presupuestales en ministerios del área social como el Ministerio de Salud y el retiro de trabajadores del estado. Una vez más, la excepción fue el sector salud: bajo esta misma administración del PUSC, el seguro social comenzó a trabajar en una segunda propuesta de reforma del sector, en la cual la contratación de servicios privados no estaba contemplada. Por lo tanto, las medidas fueron precisamente contrarias a lo esperado, en tanto expandían, en lugar de reducir, el papel del estado. Es posible que la historia personal de Calderón, cuyo padre había sido el fundador del seguro social en los años 40, haya incidido en las medidas sectoriales promovidas durante su gobierno. Más allá de esto, sin embargo, tal como lo muestro más abajo, la reforma de la salud no fue producto de esta administración y este partido, sino de iniciativas provenientes del seguro social que, si bien no deben considerarse apartidarias, sí fueron fuertemente bipartidarias.

Hasta 1993, la oposición a la contratación externa de servicios dentro del propio seguro social provenía de cuadros tecnoburocráticos y se reflejó, por ejemplo, en un conjunto de documentos de la gerencia médica (Dirección de Servicios Médicos de Atención). De acuerdo con estos estudios, la relación costo-efectividad de las cooperativas era alta. Sin embargo, se argumentaba que los costos absolutos no solo eran mayores que en clínicas equivalentes administradas por el seguro social, sino también excesivos para ser replicados. Por tanto, los estudios revelaban que era inviable financiar un servicio similar a otras personas y, por lo tanto, 
contradecía los principios de universalidad, equidad y solidaridad del seguro social.

Esta crítica que databa ya de varios años, solo con el inicio de negociaciones con el Banco Interamericano de Desarrollo - BID tuvo capacidad propositiva mediante el acceso a una alternativa reformista. En efecto, la propuesta fue promovida durante negociaciones que las autoridades costarricenses llevaron a cabo a lo largo de 1993 con el BID y que culminarían con la aprobación de una solicitud de préstamo por parte de la Asamblea Legislativa en 1994. Con esta propuesta, quedó descartada la posibilidad de que la reforma de la salud costarricense incluyera un componente relevante de privatización y descentralización de servicios.

En las negociaciones durante las cuales se negociaron los préstamos, primero con el BID y enseguida con el Banco Mundial, tuvieron un papel muy importante cuadros técnicos del seguro social pertenecientes a ambos partidos políticos. Tal fue el caso, por ejemplo, del Dr. Álvaro Salas, entonces director de la Dirección de Servicios Médicos de Atención y presidente ejecutivo de la Caja bajo la siguiente administración del PLN (Figueres, 1994-1998). El PLN asumió el gobierno en 1994, año de aprobación del préstamo y, sin embargo, dada la consulta bipartidaria durante la elaboración del proyecto, tanto técnica como política, la alternancia partidaria no supuso mayores cambios. Finalmente, cabe señalar que ninguno de los dos partidos reclamó, al menos públicamente, ser 'dueño' de la política de reforma. ${ }^{12}$

En suma, en relación a las políticas sectoriales examinadas, las preferencias políticas expresadas en los discursos partidarios no constituyen, por sí solas, un factor explicativo suficiente para dar cuenta de la emergencia, contenido y resultados de cada uno de los intentos reformistas. Por el contrario, el caso examinado indicaría que aquello que los partidos políticos propugnaron como lineamientos generales de reforma no se correspondió con sus preferencias ideológicas y/o medidas promovidas en otros sectores sociales. Al mismo tiempo, lejos de que los partidos políticos estuvieran ausentes, las negociaciones entre partidos alimentaron acuerdos al interior del propio sector, generando lo que podríamos llamar el efecto neto-partido en la emergencia, contenido y resultados de las reformas.

\section{Las políticas reformistas como respuesta a demandas de las instituciones financieras internacionales}

Habiendo examinado el papel de los partidos políticos en las políticas reformistas, considero ahora el papel de las Instituciones Financieras Internacionales - IFIs, particularmente el Banco Mundial y el BID, en tanto actores internacionales centrales en los 
procesos de reformas en curso desde el mismo inicio del proceso de ajuste estructural en la región. En términos simples, la pregunta a contestar es en qué medida los intentos de reforma de la salud costarricense se debieron a demandas o presiones de las IFIs. Desde los años 80, las IFIs han tenido una creciente importancia en la economía y la política costarricenses, primero a través de las medidas de ajuste y luego a través de los paquetes de reforma estructural. Podemos, por lo tanto, esperar que estas agencias hayan tenido algún papel en la emergencia, contenido y resultados de las reformas sectoriales en general, y de la salud en particular. Respecto a esto último, podemos también esperar que las IFIs proveyeran mayor apoyo a aquellas reformas que se correspondieran mejor con su propia agenda reformista y que, eventualmente, usaran mecanismos de presión para ejecución y continuidad doméstica. ${ }^{13}$

A los efectos de examinar estas hipótesis cabe tener en consideración al menos tres aspectos: la especificidad de las transformaciones en las políticas sociales (versus las más estudiadas políticas macroeconómicas); los múltiples mecanismos en juego en el intercambio entre las IFIs y los países (versus el supuesto de que la condicionalidad es, bien el único mecanismo existente, bien condición suficiente para la implementación de políticas domésticas) y variaciones en las políticas promovidas por las IFIs a lo largo del tiempo (versus la noción de que las IFIs tienen una y solo una agenda, prácticamente invariable a lo largo del tiempo).

En primer lugar, el papel de las IFIs en relación a las políticas sectoriales (y no solo macroeconómicas) ha sido aún poco estudiado. Dentro del paquete de reformas estructurales, inicialmente las IFIs consideraron la reforma social como subsidiaria a los objetivos macroeconómicos, al tiempo que extendían a este sector la misma prescripción privatizadora y descentralizadora propuesta al sector público en su conjunto. Fue a partir de la consideración macroeconómica de la política social que las IFIs en general, y en particular el Banco Mundial, comenzaron a promover reformas institucionales en el sector social. ${ }^{14}$

El escenario del primer intento de reforma del sector salud costarricense fue la negociación de un paquete de medidas de ajuste estructural entre el gobierno y el Banco Mundial. ${ }^{15}$ El Programa de Ajuste Estructural - PAE costarricense, al igual que aquellos firmados en otros países de la región en ese tiempo, consistía en un préstamo orientado a la transformación macroeconómica del país. Las políticas sociales fueron parte del programa solo en tanto y en cuanto la reducción del déficit fiscal requería de una reducción del gasto social. Las IFIs no participaron ni del diseño ni del financiamiento de reformas sectoriales, sino que tuvieron como papel principal la aprobación del PAE y el cumplimiento de sus líneas generales. Sin embargo, cabe señalar, que ya en el marco del 
PAE, el Banco Mundial financió asistencia técnica destinada a elaborar un primer diagnóstico de políticas sociales. ${ }^{16}$

En ese marco, el gobierno nacional priorizó el sector salud antes que a otros sectores de política social por una razón simple: el sector salud era el que estaba más cerca de mostrar avances en relación a nuevas modalidades de prestación de servicios. En efecto, si bien había sido iniciada por otros motivos, la contratación de empresas privadas para la provisión de servicios que la CCSS se encontraba considerando en ese momento, correspondía a las medidas de privatización y descentralización que promovía el Banco Mundial. Para el gobierno costarricense, el mostrar este programa fue tan conveniente como accidental fue el que su debate correspondiera a la negociación del PAE. Cuando presionado a mostrar resultados de transformación del sector social, el gobierno usó la contratación de terceros como evidencia de su voluntad de promover reformas estructurales en el sector social.

En marcado contraste, en el segundo intento de reforma de la salud, el Banco Mundial y el BID participaron de la formulación de la política de reforma, al tiempo que se involucraban de lleno con la elaboración de una agenda social (World Bank, 1993). Esta última trascendía los aspectos de infraestructura (previamente línea prioritaria del BID) y de ajuste macroeconómico (arena principal de reformas del Banco Mundial luego de la crisis mexicana de 1982). No solo dicha agenda se amplió, sino que los bancos promovieron la elaboración teórica y sistematización de evidencia empírica en relación a aspectos institucionales y programáticos propios del sector. ${ }^{17}$

Cabe señalar que hasta el inicio de las políticas de ajuste estructural, las agencias internacionales más importantes en el financiamiento, diseño e implementación de políticas de salud eran específicamente sectoriales. Tal fue el caso de la Organización Panamericana de la Salud - OPS, en los años 70 en Costa Rica y en América Latina, especializada en atención primaria. ${ }^{18}$ La Organización Internacional del Trabajo - OIT, por su parte, tuvo un papel protagónico en relación a los programas de medicina curativa localizados en los seguros sociales y asistió técnicamente a Costa Rica durante varias décadas. ${ }^{19}$

A través de los años 80, el Banco Mundial y el BID expandieron sus departamentos especializados en política social, número de especialistas y publicaciones en el área. Hacia mediados de los años 90, estas IFIs ya lideraban el diseño, financiamiento y asistencia técnica en materia de reforma social. OPS, contrariamente a los años 70 cuando sus técnicos eran la mano derecha de las autoridades sanitarias costarricenses y tenían un importante peso en la definición de las políticas, vieron restringido su ámbito de participación a aspectos programáticos específicos. ${ }^{20}$ Mientras tanto, 
las IFIs se convirtieron en proveedores de marcos generales de reforma, agencias sectoriales. ${ }^{21}$

En este escenario, Costa Rica negoció un préstamo especialmente destinado a transformar el sector salud. De hecho, el propio préstamo fue producto de los nuevos tiempos que corrían en las IFIs: la propuesta surgió durante la negociación de recursos destinados a infraestructura de la CCSS y del Ministerio de Salud..$^{22}$ Fue de las propias misiones del BID la recomendación de que Costa Rica promoviera una reforma institucional a partir de un préstamo conjunto, BID/Banco Mundial. Las autoridades costarricenses estuvieron de acuerdo y comenzaron a trabajar en la propuesta (Salas, 1997). ${ }^{23}$ Además del emergente interés y participación de las IFIs en el sector, esta negociación mostró una coordinación novedosa entre las agencias. ${ }^{24}$

Una vez establecida la centralidad y el cambio en el papel de las IFIs en los intentos reformistas del sector salud costarricense, cabe examinar los mecanismos predominantes de relaciones entre las IFIs y las elites costarricenses. La literatura ha examinado considerablemente el papel de las presiones externas en las políticas económicas latinoamericanas (Kahler, 1989), sin embargo, no así su papel en las políticas sociales y, en particular, las reformas institucionales de estas políticas. Adicionalmente, cuando las IFIs han sido consideradas en el estudio de las políticas económicas, lo han sido principalmente en tanto inductoras de políticas a través del llamado policy-based lending (Wilson, 1998). Ciertamente, no puede haber dudas de que las IFIs han usado el crédito y la ayuda financiera para inducir políticas. Más aún, en el caso de Costa Rica, en la primera mitad de los años 80, las IFIs, en particular el FMI, la Usaid y bancos privados, usaron la condicionalidad cruzada como un mecanismo de presión. Sin embargo, no es claro que éste haya sido el único mecanismo relevante de relaciones entre las IFIs y las elites domésticas. Al mismo tiempo, en los países en desarrollo, dificultades técnicas e institucionales le han hecho difícil a las IFIs el monitoreo de los procesos de implementación de políticas en los países en desarrollo (Morsley, 1987). Por ello, en general, pareciera que la condicionalidad tiene una limitada capacidad de predicción del cumplimiento de políticas (Haggard, 1986).

Una perspectiva más amplia de la relación entre las IFIs y los países que atraviesan procesos de ajuste estructural señala al menos tres mecanismos en juego en la interacción entre las agencias y los procesos domésticos: la inducción por parte de las agencias internacionales, la adaptación resultante de la imitación entre países y el aprendizaje, resultante de la acumulación de conocimiento relevante para la comprensión de las políticas y sus efectos (Ikenberry, 1990). Por lo tanto, reconociendo la importancia de las relaciones de poder e interés como factores que están detrás de la 
formación de políticas, este análisis enfatiza la importancia de las ideas y su transformación a lo largo del tiempo. Si no más, este análisis hace una contribución importante, la cual es no asumir sino establecer empíricamente la relación entre las agencias internacionales y los países.

En este marco cabe señalar que el primer intento reformista de la salud costarricense tuvo un papel de corto plazo en las negociaciones entre el gobierno y el Banco Mundial. Estrictamente, no fue una política inducida por ninguna de las IFIs sino una política que el gobierno utilizó instrumentalmente frente a las IFIs para los efectos de mostrar su compromiso con el proceso de ajuste estructural. Así lo muestran los tiempos de estas políticas: las cooperativas médicas comenzaron antes que el Banco Mundial demandara el rediseño de la política social. Por su parte, la segunda reforma de la salud fue cualquier cosa menos impuesta a Costa Rica. Por el contrario, la nueva agenda de reforma que emergía de la iniciativa del BID venía a resolver la confrontación doméstica generada alrededor de la contratación privada de servicios propia de la primera reforma.

El estudio de las negociaciones de los tres PAEs costarricenses muestra que las elites costarricenses tuvieron importante dosis de discrecionalidad para manipular las opciones de política, tanto en la arena nacional como internacional para los efectos de promover su propia agenda (Raventós, 1996). Más concretamente, las elites costarricenses utilizaron el argumento de la 'viabilidad doméstica' de las políticas como forma de incrementar su capacidad de negociación con las IFIs acerca del contenido de la reforma, tanto como arguyeron 'presiones internacionales' a los efectos de legitimar decisiones en el ámbito doméstico. En este sentido, la instrumentalidad de las elites domésticas parece haber sido bidireccional, es decir, hacia las IFIs y hacia el ámbito doméstico. ${ }^{25} \mathrm{~A}$ su vez, a lo largo de dicha negociación, las elites costarricenses pudieron apoyarse en los logros sociales previos del país, tales como el logro de la segunda mortalidad infantil más baja de la región y una esperanza promedio de vida similar a la de países del primer mundo. Es de esperar que estos logros le hayan dado a Costa Rica mayores márgenes que la de otros países de la región (Cercone, 1996).

Más aún, tiene sentido especular acerca del carácter de 'laboratorio' que tiene Costa Rica y el interés de las IFIs de experimentar con medidas de mejoramiento de la relación costoefectividad en tanto sistema de salud predominantemente público. Bien puede ser el caso que al financiar una reforma, las IFIS y sus técnicos hayan perseguido objetivos de experimentación y elaboración de teoría organizacional para reformas futuras, tanto o más que los objetivos concretos de esta reforma en particular. La plausibilidad de esta hipótesis tiene todavía más sentido si 
pensamos: primero, en la escala de inversiones que un pequeño país como Costa Rica requiere para promover transformaciones institucionales, aunque sean de gran envergadura; segundo, en la reputación de contar con sólidos cuadros técnicos y una alta capacidad institucional; y tercero, el perfil epidemiológico de 'primer mundo' de la población, en particular medidos en términos de esperanza de vida y causas de muerte. Combinadas, estas características le permiten a Costa Rica ser un caso atractivo del cual extraer lecciones que a su vez trasciendan a los propios países de la región.

La política hacia la atención primaria ofrece un buen ejemplo. En este caso la controversia era respecto a si el nivel primario de atención debía incluir también medicina curativa y con qué grado de especialización. Mientras el Banco Mundial argumentaba que el paquete de servicios debía ser relativamente restringido a medicina preventiva y primaria, los técnicos costarricenses argumentaban la necesidad de un paquete comprensivo que incluyera servicios especializados. El argumento era que si bien esta medida inicialmente dispararía la demanda e incrementaría los costos de los servicios, luego alivianaría la presión sobre los más costosos servicios hospitalarios. Finalmente, el paquete reflejó una transacción de las dos partes, la cual permitiría una dilucidación empírica de la hipótesis de rediseño institucional sostenida por los técnicos costarricenses. En este sentido, el componente de aprendizaje tiene un papel interesante a la hora de establecer la racionalidad de las IFIs en apoyar una reforma que, en términos generales, contradice sus expectativas respecto al papel del sector público en la prestación directa de servicios.

Una distinción entre el primer y el segundo intentos de reforma de la salud, considerados en este trabajo, tiene que ver con la naturaleza del compromiso establecido en cada caso entre las IFIs y el país. La primera política reformista fue formulada dentro de un marco general de transformaciones macroeconómicas y objetivos vagamente definidos en relación al sector social. La segunda política reformista, sin embargo, supuso un cambio específicamente sectorial, cuyos objetivos, componentes y acciones estaban contenidos en la solicitud de préstamo. ${ }^{26}$ Esto podría explicar por qué el Banco Mundial ‘toleró' vetos domésticos, tanto políticos como institucionales, a la contratación externa propia de la primera reforma, pero aseguró la continuidad de otra que promovía crecimiento y consolidación de la participación estatal en el financiamiento, gerenciamiento y entrega de servicios de salud. En realidad, las IFIs no necesariamente contaban con mecanismos para 'empujar' la continuidad del primer intento reformista y, sin embargo, sí los tenían en el segundo. La paradoja del asunto es, evidentemente, el que las IFIs carecieran de capacidad de inducción 
en una reforma inspirada en reducción del aparato del estado y no en una que aumenta dicha participación, tanto en materia de cobertura como de seguimiento y evaluación.

Finalmente, las agendas promovidas por las IFIs desde la mitad de los 90 han mantenido constantes sus principios orientadores, pero transformándose en sus medidas, de aquellas más ortodoxas centradas en la privatización de la prestación de servicios a otras más preocupadas con la reorganización del sector público (sea que supongan o no privatización y descentralización). ${ }^{27}$ En un principio, las IFIs no solo apoyaron sino que presionaron al gobierno costarricense a llevar a cabo medidas generales de privatización y descentralización de servicios públicos. Más tarde, sin embargo, y tal como se refleja en el informe anual del Banco Mundial de 1993, las agencias mostraron interés en reformas que incorporaran arreglos organizacionales y mecanismos de mercado dentro del propio sector público (World Bank, 1993).

La perspectiva del Banco Mundial respecto al sector público fue evolucionando hasta contar con una teoría organizacional del cambio en el sector público. Esta agenda, tal como es compartida por el Banco Mundial y el BID, se resumiría años después en Beyond the Washington Consensus: institutions matter (World Bank, 1998). En este contexto, el énfasis en la privatización y descentralización de servicios ha sido reemplazado por la preocupación más general acerca de cómo mejorar los arreglos contractuales al interior del propio estado. Esta agenda tal como la conocemos hoy fue elaborada con insumos de las experiencias reformistas de la primera mitad de la década. Cuando Costa Rica firmó el acuerdo de préstamo para la reforma sectorial de la salud, esta agenda estaba menos elaborada que lo que lo está hoy, pero sin embargo, ya estaba instalada. Es posible además que el sector salud haya provisto insumos teóricos y empíricos para la reformulación de la agenda social en general.

Arriba he argumentado que las IFIs muchas veces tienen una capacidad relativamente escasa de inducción de políticas. En segundo lugar, he argumentado que no siempre la imposición de políticas es el principal objetivo de las IFIs sino que, muchas veces, su principal objetivo es la elaboración de dichas políticas mediante, precisamente, la ejecución de reformas domésticas. En otras palabras, pareciera apropiado poner mayor atención al accionar de las agencias internacionales más allá de su poder de inducción de ciertas agendas, en tanto generadoras de agendas y, en general, componente central para nuestra comprensión del papel del conocimiento en las reformas actuales. Antes de explorar este punto, primero me refiero al papel de los factores políticos e institucionales propios del sector salud en explicar la suerte de los intentos reformistas. 


\section{Las políticas reformistas como resultante de intereses sectoriales}

Con el objetivo de explicar las reformas de la salud, hasta ahora he argumentado que éstas no tuvieron una correspondencia directa con las preferencias ideológicas históricas de los partidos políticos, ni respondieron meramente a presiones internacionales. Del análisis de factores explicativos propios del ámbito nacional e internacional, abordo ahora uno de carácter sectorial. Es posible que las configuraciones sectoriales de poder e interés en algún grado expliquen los vetos y apoyos recibidos por las respectivas políticas de reforma. Se trata, en suma, de examinar la hipótesis de que el diseño y la adopción de los intentos reformistas en gran medida se explican en función de su generación de vetos y coaliciones dentro del propio sector salud.

Como ya ha sido establecido más arriba, de hecho, ambos intentos reformistas analizados aquí surgieron a partir de la iniciativa de las autoridades políticas y cuadros tecnoburocráticos sectoriales. En efecto, a través de los años 80 y 90, las autoridades sectoriales políticamente designadas en la CCSS y el Ministerio de Salud, profesionales de la salud con carreras institucionales reconocidas, tuvieron a su cargo la iniciativa y conducción de dichas reformas. ${ }^{28}$ Por lo tanto, la fuente de legitimidad en el puesto de dichas autoridades no fue solo el apoyo político partidario, sino también una reconocida trayectoria institucional.

A su vez, los cuadros técnoburocráticos superiores del seguro social, en su mayoría funcionarios de carrera, proveyeron los insumos técnicos e institucionales para llevar estas reformas adelante. Por ende, las políticas de reforma se llevaron adelante fundamentalmente por parte de los cuadros técnicos existentes y no por parte de equipos técnicos ad hoc. Más aún, Costa Rica parece haber atravesado la estabilización y el ajuste con una menor concentración en el poder ejecutivo que en otros países de la región, en los cuales las autonomías sectoriales fueron fuertemente debilitadas a lo largo del proceso. En Costa Rica, por el contrario, a lo largo del período considerado, las instituciones sectoriales mantuvieron importantes grados de autonomía, aunque no insularidad político partidaria. ${ }^{29}$

Dicho esto, es importante señalar que en el primer y segundo intentos reformistas, el papel de las elites tecnoburocráticas en el diseño de las reformas fue diferente. El primer intento fue promovido por médicos del seguro y apoyado, luego de vencer reticencias iniciales, por el entonces presidente ejecutivo de la Caja. La iniciativa y el equipo propulsor de la política fueron en cierto grado ad hoc con las instancias institucionales correspondientes. De hecho, la política carecía del apoyo y, más aún, fue opuesta por la mayoría de 
los cuadros tecnoburocráticos del seguro social (Miranda, 1994; Sáenz, 1996; Vargas, 1994). En este sentido, el diseño de la reforma evidenció una cierta dosis de bypass institucional.

La implementación, por su parte, comenzó de inmediato, mostrando un importante grado de disciplina por parte de los cuadros superiores del seguro social. Sin embargo, dado que muchas medidas giraron en torno al servicio creado, la cadena de mando jugó un papel relativamente marginal. Asimismo, los cuadros técnicos superiores del seguro social pronto mostraron su desacuerdo con la reforma, no tanto mediante un bloqueo en su ejecución, sino cuestionando la viabilidad y conveniencia de los resultados de la política, sus implicancias financieras en el largo plazo y su conflicto con los principios orientadores del sistema.

En efecto, el veto tecnoburocrático a las cooperativas médicas se mostró en un conjunto de estudios, los cuales, si bien reconocían el alto grado de costo-efectividad de las cooperativas, señalaban su excesivo costo global. ${ }^{30}$ Con el primer punto se reconocían los logros de las cooperativas; con el segundo se argumentaba la inviabilidad financiera de extender la experiencia a otras comunidades. De esto se concluía que la contratación de terceros estratifica los servicios y por ende amenazaba el principio de equidad del sistema (Zamora \& Sáenz, 1995). La divulgación de estos estudios acentuó las tensiones ya existentes entre las cooperativas y los cuadros del seguro social. Sin negar de la honestidad de estos planteos, cabe también especular respecto a la percepción de amenaza a su centralidad en la administración de servicios del seguro que pudieron sentir los cuadros técnoburocráticos del seguro social.

Por su parte, los gerentes de las cooperativas frecuentemente hicieron ellos mismos un bypass a los mecanismos institucionales del seguro social, resolviendo problemas a través de contacto directo con las autoridades o comisiones especialmente creadas para tales fines, evitando así los canales institucionales de control y coordinación establecidos para el conjunto de las clínicas del país (Vargas, 1994; 1996). Estos mecanismos ad hoc, en parte alimentados por el conflicto con los cuadros técnicos del seguro social, al mismo tiempo profundizaron dicho conflicto dando pie a acusaciones de trato clientelista y preferencial a las cooperativas por parte de la Junta Directiva de la Caja (Durán, 1995; 1997).

Lo que en realidad disparó el segundo intento reformista fue el acceso de los cuadros técnicos costarricenses a una nueva alternativa reformista a la que adhirieron no solo los opositores a la contratación de terceros, sino también los promotores de las cooperativas. En efecto, esta segunda política reformista fue diseñada por un equipo de técnicos del seguro social al cual se sumaron consultores externos entre quienes estuvieron los propios promotores de la creación de cooperativas médicas. ${ }^{31}$ De hecho, la participación 
de estos gerentes en el diseño de la segunda reforma por un momento sugería que las cooperativas adquirirían el carácter de laboratorio de la reforma nacional en ciernes, solo que reemplazando la prestación privada por la prestación pública.

En suma, la principal diferencia entre el primer y el segundo intento reformistas fue que el primero careció del apoyo tecnoburocrático. A pesar de esto, las negociaciones macroe-conómicas del país con las IFIs en marcha en ese momento generaron condiciones para su aprobación e implementación. El segundo intento fue motivado cuando cuadros técnicos opuestos al primer intento reformista vislumbraron una alternativa. Esta alternativa reformista no solo era técnicamente sólida sino que, estratégicamente, proveía de una mejor oportunidad para la generación de consensos al interior del seguro social, removiendo vetos que habían frenado el primer intento reformista. Cabe preguntarse acerca de cómo esta segunda alternativa reformista llegó a ser tal para las elites costarricenses. Como veremos más abajo, el pasaje de uno a otro intento reformista estuvo fuertemente dado por la existencia, en el ámbito internacional, de una alternativa hasta entonces inexistente.

\section{Las políticas como resultado de la imitación selectiva de ambientes internacionales}

Como veremos, la disponibilidad de agendas de reforma del sector salud en uno y otro momento tuvo un papel importante para explicar el comportamiento de los actores en torno a los intentos reformistas domésticos, en este caso en Costa Rica. En efecto, más allá de los intereses de los actores involucrados, sectoriales, nacionales e internacionales, aquí examino la hipótesis de que, a la hora de explicar el curso de las reformas, las agendas disponibles tienen un peso explicativo propio. Asimismo, dichas agendas no pueden considerarse en términos estrictamente domésticos, sino que se inscriben en ambientes internacionales de los cuales las elites domésticas adoptaron y adaptaron estas agendas.

En tanto las organizaciones son imitadoras de las acciones que llevan adelante organizaciones similares ('pares') en el ámbito internacional, los ambientes internacionales sectoriales son la fuente por excelencia de prescripciones de acciones deseables y posibles (DiMaggio \& Powell, 1983). Es precisamente en el marco de transformaciones en el menú de reformas disponibles en dicho ambiente internacional, que los intentos reformistas del sector salud costarricense comenzaron a llevarse adelante, mucho antes de que los partidos políticos o las instituciones financieras internacionales tuvieran la iniciativa de promover dichas reformas. 
En efecto, antes de los partidos políticos o que las IFIs tuvieran cualquier participación en la reforma costarricense, las autoridades sectoriales costarricenses iniciaron un proceso de revisión de los arreglos organizacionales existentes. Este proceso fue en gran medida alimentado por la exposición de las autoridades de la CCSS al sistema inglés y al intercambio de asesoramiento e ideas entre técnicos de ambos países (Miranda, 1994). En la segunda mitad de los años 80, consultores ingleses visitaron Costa Rica, proveyendo de insumos técnicos que le permitieron a Costa Rica pensar en opciones de arreglos organizacionales que no habían sido considerados hasta entonces (Miranda, 1993). Sin embargo, como veremos, la adopción de dichos arreglos no fue mecánica.

El alto grado de exposición e influencia internacional no debería sorprender: ningún país produce políticas en forma aislada de otras partes del mundo. Las autoridades de la salud y los cuadros superiores del seguro social costarricense y, en general latinoamericano, han estado históricamente abiertos a las experiencias de otros sistemas nacionales. La propia creación del seguro social costarricense en los años 40 fue resultado de lecciones extraídas del modelo chileno y luego de otros sistemas como el mexicano. En general, el intercambio formal e informal de ideas y experiencias ha tenido un papel central en la formación de políticas. Algunas veces, dicho intercambio fue establecido directamente entre los cuadros tecnoburocráticos; en otros, mediado por agencias internacionales como la ILO y la OPS. Solo una vez que las reformas estructurales comenzaron, las IFIs comenzaron a tener un papel en la divulgación de experiencias domésticas similares a las de aquellas agencias, aunque mediante otros mecanismos.

En el primer intento reformista, la adopción de arreglos novedosos fue hecha en forma experimental. A mediados de los años 80, médicos del seguro social y del Ministerio de Salud se encontraban retornando a Costa Rica habiendo completado sus estudios de postgrado en administración pública de servicios fuera del país. Dos de ellos, los doctores Mauricio Vargas y Fernando Marín, habían terminado sus estudios en administración de la salud en Colombia (Universidad Javeriana) y lideraron la elaboración de propuestas de transformación organizacional. A varios años de experiencia trabajando en esas agencias, tanto Vargas como Marín, agregaron su entrenamiento de postgrado en gerenciamiento y entrega de servicios de salud. En términos generales, pues, la experimentación con la terciarización de servicios no surgió de una tendencia neoliberal sino, por el contrario, en el marco de compromiso socialdemócrata con la medicina pública y la seguridad social. Hay que tener presente que al hacer estas afirmaciones ambos profesionales tenían un doble respaldo. Primero, estaban desarrollando una experiencia en una de las comunidades más 
populosas y socio económicamente heterogéneas de la ciudad capital de Costa Rica. Segundo, al momento de realizarse las entrevistas contaban con el apoyo de organizaciones comunitarias que habiendo sido en un inicio críticas y sospechosas de la dirección neoliberal de la propuesta, posteriormente dieron todo su apoyo a la cooperativa.

La experimentación buscó combinar medicina preventiva y curativa (la cual, se esperaba, combinara una mejora de la calidad de la atención con una disminución de los costos) $)^{32}$ y la descentralización en materia de programación y entrega de servicios, de forma de mejorar la adecuación de la oferta a las necesidades de la población. Respondiendo a estas tendencias, las autoridades del seguro social promovieron innovaciones en clínicas ubicadas en el área metropolitana de San José. ${ }^{33}$ Ningún intento de innovación institucional llegó muy lejos y quienes estuvieron a su cargo arguyeron que el cuello de botella había sido el modelo administrativo de provisión de servicios, con sus limitaciones en materia de toma de decisiones y contratación de personal a nivel local. La alternativa fue entonces la contratación externa de servicios, finalmente aprobada en 1988 con la creación de una clínica en Pavas, Coopersalud, y profundizada con la aprobación de otras dos clínicas en 1990 y 1992 (Coopesain y Coopesana, respectivamente). El motor de la reforma fue, pues, fuertemente sectorial y apoyada, por parte de autoridades y técnicos del sector salud, en la adopción y adaptación de políticas disponibles internacionalmente.

Las alternativas reformistas prevalecientes en el ambiente de política del sector salud fueron aquellas promotoras de medidas a favor de la privatización y la descentralización de servicios. Estas medidas contradecían el modelo existente en Costa Rica, altamente público y organizado con base en arreglos organizacionales jerárquicos. En ese momento, pues, el foco de la selectividad de la política llevada adelante por las elites costarricenses fue el tipo de agente externo a contratar. Más específicamente, se trató de evitar contratar organizaciones típicamente privadas. Los llamados prestadores privados de servicios fueron cooperativas, con lo cual el seguro social amortiguaba la generación de ganancias como único motor o incentivo organizacional. Asimismo, dichas cooperativas fueron creadas dentro de un marco regulatorio público que, al menos formalmente, les requería participar de estrictos mecanismos de control y coordinación administrativos, propios del seguro social en su conjunto.

En el segundo intento reformista, la alternativa reformista prevaleciente en el ambiente internacional de políticas de salud cambió de un eje público/privado a otro administración burocrática/ competencia regulada entre servicios públicos. En este caso, la selectividad de las elites costarricenses se expresó en su imitación 
de medidas destinadas a expandir la intervención pública. En esta nueva agenda adquirieron centralidad temas como el de los mecanismos de asignación de recursos (de mercado antes que burocráticos) y el tipo de servicios a proveer (preventivos y de atención primaria antes que curativos). En Costa Rica, la elaboración de esta clase de propuesta de reforma requirió más de un año de trabajo. A través de ese tiempo, cuadros superiores del seguro social, en su mayoría médicos, estudiaron y conocieron las experiencias regionales y europeas, así como algunas de las lecciones domésticas de años previos (Ayala, 1996).

En la búsqueda de medidas que llevaran adelante reformas al sector salud costarricense, la premisa de las elites fue el mantenimiento de los principios de universalismo, equidad y solidaridad del sistema. Para ello, la región no ofrecía un camino exitoso de reforma que mantuviera los principios que Costa Rica quería mantener. En particular, Chile aparecía como el modelo dominante de reforma en América Latina. Sin embargo, la reforma chilena de principios de los años 80 contradecía los principios orientadores de transformación del seguro social costarricense, dado que instituía dos sistemas paralelos, uno de mercado para quienes podían pagarlo y otro público para los pobres. ${ }^{34}$ Además, los cambios epidemiológicos de la población costarricense (posterior a la transición) y su nivel de gasto (cercano al 8\%) distanciaban a Costa Rica del resto de la región y lo acercaban, al menos en la percepción de sus propias elites, a los sistemas europeos. Es por ello que Europa y no América Latina fueron las principales fuentes de búsqueda de ejemplos de políticas de transformación.

En este marco, las autoridades costarricenses se interesaron principalmente por el caso inglés y su sistema nacional de salud, de alcance universal y financiado con fondos públicos. A mediados de los años 80, cuando Costa Rica comenzó a explorar alternativas de reforma, el menú reformista disponible al momento del primer intento era muy limitado. El sistema de 'capitación' era una de las pocas opciones para la asignación de recursos que no supusiera privatización del financiamiento del sistema como sí lo había hecho Chile. En marcado contraste, el segundo intento reformista tuvo lugar en un momento en que el menú de opciones había incorporado una segunda avenida reformista, incluso contemplando medidas que promovían la expansión y no la disminución de la participación estatal en el sector. Las autoridades costarricenses buscando opciones de reforma en este segundo momento percibieron medidas emanadas de la corriente de nuevo gerenciamiento público como aquellas más apropiadas para reorganizar, sin amenazar, el seguro social costarricense. En otras palabras, en este segundo momento, las elites costarricenses tenían acceso a una agenda que era mucho más apropiada para la configuración política e 
institucional que se proponían transformar que en el primer momento.

En cada caso, como lo documentan los numerosos viajes realizados durante 1991 y 1992 a países como Chile, España y Suecia, los reformadores costarricenses buscaron en el ámbito internacional sectorial, alternativas de política adecuadas para su uso doméstico. Dicha búsqueda no fue, por tanto, indiscriminada sino altamente selectiva. Las elites técnicas y políticas del sector se volvieron a aprender de países con los cuales Costa Rica había tenido relaciones previas de cooperación (como Suecia), países que percibió como afines y con los cuales en ese momento tenía relaciones de asistencia técnica (como Inglaterra), o países que el Banco Mundial se encontraba promoviendo como ejemplares (como España). Además, la búsqueda rechazaba países que se consideraron ejemplo de lo que no se quería hacer (como Chile) (y aunque años después cuadros técnicos costarricenses hubieran relativizado este punto y señalado que en realidad se podía aprender de algunos aspectos gerenciales específicos de la reforma chilena). En cada uno de estos casos, las elites costarricenses identificaron aquellas medidas 'que funcionaron' y que correspondían a los parámetros domésticos de lo que debía ser cambiado o mantenido (Salas, 1996; Ayala, 1996; Cercone, 1996; Barths, 1997).

Por lo tanto, en lugar de una mera convergencia internacional de políticas que algunos autores señalan que se estaría produciendo a partir de la generalización de la imitación como mecanismo de formación de políticas, las reformas examinadas aquí indican que estamos frente a una combinación de convergencia y diferenciación. Más concretamente, este doble movimiento de convergencia y diferenciación se produce en Costa Rica a través de lo que llamo la imitación selectiva de menús de política disponibles interna-cionalmente. ${ }^{35}$ Estos mecanismos, lejos de generar una pura y simple indiferenciación de políticas, promueven movimientos simultáneos de homogeneización y diferenciación entre realidades domésticas.

En la primera reforma, la selectividad explica la clase de privatización y descentralización con la cual se experimentó. Durante la primera reforma, los arreglos institucionales adoptados para llevar adelante la privatización y descentralización fueron heterodoxos. En el segundo intento, la selectividad se tradujo en la mezcla de lecciones extraídas de varias fuentes, tales como Inglaterra, España, Suecia y Chile. De esta forma, Costa Rica buscó combinar aspectos propios de la asignación de recursos británica en servicios curativos del primer nivel de atención, con el modelo sueco de atención primaria, la competencia regulada entre hospitales llevada a cabo en España y los sistemas de información chilenos. Los criterios para elegir un país u otro como fuente de imitación, varían. 
Varios caminos 'llevan a Roma' pero todos tienen en común que la extracción de lecciones tuvo lugar en el marco de un ambiente dado de políticas sectoriales (Rose, 1991).

Sin ignorar que las IFIs han tenido un papel importante en explicar la emergencia, contenidos y resultados de las reformas costarricenses, las políticas examinadas en este trabajo indican que las elites costarricenses han tenido importantes grados de libertad en la definición de las reformas. Al mismo tiempo, sin negar el importante grado en que poder e intereses han guiado el comportamiento de estos actores, es importante considerar el papel que las elites costarricenses han tenido en la búsqueda, imitación y adaptación de políticas disponibles internacionalmente. Sin que esta búsqueda haya estado guiada por una perfecta racionalidad instrumental, las elites costarricenses tuvieron un importante grado de elección y rechazo de políticas sectoriales, todas éstas consideradas legítimas y deseables dentro del ambiente internacional de reformas sectoriales ${ }^{36}$ En suma, ninguno de los dos intentos reformistas, y por lo tanto la suerte que corrieron, puede ser comprendido sin reconocer el fuerte papel jugado por las elites domésticas. Sin embargo, la consideración de este papel en la formación de las políticas sociales adquiere un nuevo significado cuando se lo considera en el marco de ambientes sectoriales internacionales dentro de los cuales operan las decisiones domésticas.

En suma, examinar el accionar de las administraciones nacionales, las agencias financieras y los actores sectoriales simultáneamente, es condición necesaria para entender los vetos y apoyos de cada intento reformista. Al mismo tiempo, a los efectos de explicar el diseño y la adopción de dichas reformas, dicho análisis de actores debe combinarse con un análisis de agendas disponibles para su actuación. La disponibilidad de modelos sectoriales para promover reformas en uno y otro momento, y su grado de adecuación a las configuraciones domésticas iniciales a las reformas, complementan la explicación.

\section{El procesamiento doméstico de ambientes internacionales de política sectorial}

El objetivo de este trabajo ha sido explicar la emergencia, contenido y suerte de las reformas diseñadas y adoptadas en el sector salud costarricense. La evidencia presentada sugiere, primero, que los hacedores de políticas no diseñaron estas políticas en una 'burbuja'. Por el contrario, lo hicieron en el marco de ambientes sectoriales internacionales de los cuales extrajeron alternativas reformistas y posibles medidas para llevarlas adelante. Dichos ambientes sectoriales, en tanto conjunto de teorías y experiencias, constituyen los proveedores de alternativas y medidas domésticas. Asimismo, la disponibilidad de alternativas cambia a lo largo del 
tiempo, entre otras cosas, por su permanente adopción y adaptación y por cierto mecanismo de prueba de hipótesis de diseño institucional que esto permite. Como resultado, los ambientes de política cambian y también cambia la viabilidad política de las alternativas que éstos proveen para un escenario doméstico determinado.

En segundo lugar, la evidencia presentada aquí sugiere que las elites costarricenses no actuaron meramente en función de sus intereses, sino en función de lo que podríamos denominar, el procesamiento 'interesado' de politicas disponibles internacionalmente. En otras palabras, los actores, al menos en el sector salud examinado, no ignoraron, bajo riesgo de perder legitimidad, las alternativas reformistas prevalecientes internacionalmente. Por el contrario, dichos actores procesaron estas alternativas en función de los intereses específicos en juego. Concretamente, los cuadros tecnoburocráticos de la Caja estaban interesados en mantener la línea de mando que la prestación de servicios a través de cooperativas había cuestionado. Era la organización burocrática preexistente y el control centralizado de las decisiones, la que les daba poder institucional. Por eso, cuando existieron alternativas reformistas que atendían estos intereses en forma más satisfactoria que las alternativas disponibles previamente, éstas fueron rápidamente adoptadas. Este fue claramente el caso de los cuadros tecnoburocráticos del seguro social y la emergencia de medidas orientadas a la transformación del sector público mediante, por ejemplo, la creación de cuasi mercados como alternativa a la privatización de la provisión de servicios a través de contratación de servicios privados.

En ambas reformas examinadas, las políticas fueron diseñadas mediante adaptaciones de políticas disponibles internacionalmente que permitieron hacerlas compatibles con las configuraciones de poder e intereses domésticos, tanto nacionales como sectoriales. Con base en alternativas organizacionales internacionalmente disponibles en un momento dado, las elites domésticas imitaron selectivamente aquellas medidas que mejor se correspondían con las propias. En uno y otro intento de reforma, la imitación selectiva es, más precisamente, el mecanismo mediante el cual las alternativas existentes se procesaron domésticamente.

Sin embargo, mientras que la imitación selectiva describe adecuadamente los mecanismos que entiendo operaron para la formación de las reformas de la salud, no necesariamente se aplica a otros países o a otros sectores dentro de Costa Rica. Las restricciones analíticas pueden deberse a varias razones, tales como las capacidades institucionales y técnicas domésticas existentes. En otras palabras, los mecanismos específicos a través de los cuales las elites domésticas 'filtran' los ambientes internacionales de políticas 
deberían ser objeto de investigación comparativa entre países para un mismo sector (aunque también entre períodos históricos para un mismo país).

En tercer lugar, lejos de ser estáticos, las alternativas que proveen los ambientes internacionales de política se renuevan, se amplían y restringen. A través de la década considerada en este trabajo, hubo cambios importantes que reflejaron desarrollos teóricos, aprendizajes extraídos de la ejecución de políticas, así como adaptaciones estratégicas a obstáculos enfrentados por las más ortodoxas medidas a favor de la privatización de servicios. ${ }^{37}$ Esto explica por qué las IFIs podrían, por ejemplo, apoyar la creación de mercados cuasi públicos, involucrando una expansión de la intervención estatal. Mecanismos a través de los cuales los ambientes de política en general, y las agencias internacionales en particular, desarrollan sus agendas requiere de una mayor atención fuera del alcance de este trabajo. De forma similar, se necesita más investigación acerca de las redes de política y las relaciones de poder que permiten ciertas agendas domésticas volverse parte del 'menú' ofrecido por las agencias internacionales en un cierto sector de políticas (por ejemplo, el proceso por el cual las reformas chilenas de comienzos de los 80 , y sus lecciones, se volvieron políticas ejemplificantes una vez removidas del caso particular).

El caso de Costa Rica muestra la importancia de tener en cuenta biografías y trayectorias profesionales individuales como la de James Cercone, norteamericano-costarricense, primer funcionario del Banco Mundial y luego funcionario de la Caja. Cercone fue clave en tanto 'puente' entre ambas organizaciones. Aunque la evidencia es insuficiente, podría en alguna medida dar cuenta de la trayectoria y el intercambio entre ideas domésticas e internacionales. Una línea de investigación prometedora consideraría casos nacionales seleccionados, por ejemplo Chile, Costa Rica y Colombia, para reconstruir la trayectoria de las ideas entre agencias internacionales y casos nacionales en el marco de esta 'familia' de reformas.

En cuarto lugar, la importancia de los ambientes internacionales de política no debería ser restringida ni a las IFIs ni a las políticas de ajuste estructural. Las IFIs no son las únicas agencias proveedoras de alternativas reformistas, ni tampoco las únicas en promoverlas y divulgarlas. Costa Rica estuvo expuesta a ambientes internacionales de políticas mucho antes que las IFIs participaran activamente de las reformas costarricenses. En consecuencia, la importancia relativa de las agendas internacionales en una explicación de las reformas domésticas no debería ser exclusiva de los paquetes de reforma institucional que forman parte del ajuste estructural latinoamericano. Lo que debería ser objeto de investigación empírica es, sin embargo, cuáles son los vehículos relevantes de la exposición doméstica a los ambientes internacionales 
de política en cada momento (por ejemplo, comunicación país a país, foros mediados por las IFIs, etc.).

Por supuesto, durante el período bajo análisis, otros eventos tenían también lugar, como el movimiento de organizaciones proveedoras de salud en los Estados Unidos (HMO según sus siglas en inglés por Health Maintenance Organizations), la epidemia del Síndrome de Inmunodeficiencia - Sida o la propagación del dengue, entre muchos otros. Sin embargo, los datos recogidos en el marco de la presente investigación no evidenciaron su influencia en la reforma de la Caja. En términos de las nuevas amenazas a la salud pública, es posible especular con que hayan tenido más influencia en el Ministerio de Salud, por varias décadas dedicado a la medicina preventiva y a la atención primaria, que en la medicina curativa desarrollada desde la Caja.

En suma, es necesario poner una mayor atención tanto a las restricciones como a los grados de libertad de las reformas domésticas. Más concretamente, éstas deberían entenderse en el marco de ambientes de política cuya disponibilidad de alternativas constriñe los intentos reformistas en cada momento. Estas alternativas pueden adecuarse más o menos a los intereses de las elites domésticas promotoras de reformas $\mathrm{y}$, por ende, a las condiciones iniciales, previas a la reforma de cada país. Al mismo tiempo, y al menos en casos como el de Costa Rica, cuyas elites presentan un alto grado de capacidad política, institucional y técnica, la imitación selectiva de los modelos disponibles es un mecanismo explicativo central en la adopción y adaptación de los modelos provistos por los ambientes internacionales de política a lo largo del tiempo.

El ámbito doméstico es la arena de articulación de paradigmas e intereses, tanto propiamente nacionales como supranacionales. Son el conjunto de dichos factores los ingredientes que, una vez mezclados, dan como resultado una u otra política social. Lo relevante es cómo se articulan dichos factores para dar lugar a la formación de políticas. Las relaciones de poder entre actores pueden ser de muy diverso tipo: desde la imposición, hasta la negociación y el aprendizaje. Las ideas de los cuales son portadores los actores relevantes para la formación de una política pueden reforzar, transformar o revertir dichas relaciones de poder. Por lo tanto, el papel de actores nacionales o internacionales en el ámbito doméstico tiene que ser empíricamente determinado, como lo tiene que ser el lugar lógico de las ideas.

\section{Epílogo}

Entre 1988 y 1998 Costa Rica experimentó con dos rutas reformistas de su sistema único de salud. El primer intento (1988-1994) 
enfrentó vetos políticos e institucionales que acotaron fuertemente su impacto sectorial. El segundo, iniciado en 1994, se constituyó en una ruta reformista exitosa. Ambos buscaron mejorar la calidad y cantidad de servicios bajo la égida del Seguro Social, preservando la universalidad, equidad y solidaridad de un sistema que afecta a alrededor del 90\% de la población (Mesa-Lago, 1994). Este trabajo buscó explicar la emergencia, contenido y resultado de una y otra reforma. Del análisis se desprende que el comportamiento de los partidos políticos, las agencias financieras internacionales y los actores sectoriales al momento del diseño y adopción de la reforma, solo se entiende si, al mismo tiempo, se tienen en cuenta los cambios en las agendas reformistas disponibles internacionalmente en uno y otro momento. Conjuntamente, el análisis de estos factores sugiere que el diseño y adopción de la reforma estuvo más influido por la búsqueda de legitimidad que por el ejercicio de la presión.

En términos del análisis comparado de las etapas de diseño y adopción está pendiente el fortalecer la comparación histórica siguiendo el ejemplo brindado por estudios recientes (Birn, 2005) y que deseablemente fortalezca un marco interpretativo interdisciplinario, compartido desde la historia, la sociología y la ciencia política. Por ejemplo, ¿qué tan original fue el proceso de diseño y adopción de la reforma? ¿Existe algún paralelismo entre el Banco Mundial hoy y la Rockefeller en la primera mitad del siglo veinte? ¿Hay algún punto en común entre la imitación selectiva de las reformas contemporáneas y las realizadas previamente, por ejemplo, para lidiar con la mortalidad infantil o combatir la malaria?

Además, más allá de las etapas de diseño y adopción, es preciso profundizar en la etapa de implementación, en los cambios intencionales e intencionales efectivamente producidos a partir de políticas públicas adoptadas. Entre etapas del ciclo de formación de políticas, ¿cómo cambia la lógica de formación de políticas y los factores explicativos? En el sector salud costarricense, la reforma que ha tenido lugar en los hechos ha sido bastante distinta a la definida formalmente: el sector público se ha visto debilitado en la proporción del gasto en salud, calidad y oportunidad de los servicios y se han diversificado los servicios privados, principalmente con fines de lucro aunque también cooperativo, tanto financiado con recursos públicos como 'de bolsillo'. Aunque no todo, gran parte del avance del sector privado se ha producido de maneras subterráneas y por lo tanto, perniciosas. Parte de esta oferta privada en expansión es producto de la propia pérdida de calidad y oportunidad de los servicios públicos; otra parte es producto de un debilitamiento de las fronteras entre lo público y lo privado.

La brecha de modelos entre la reforma adoptada y la reforma implementada llama la atención acerca de las distintas configuraciones existentes entre actores, paradigmas y agendas, 
nacionales e internacionales en uno $\mathrm{u}$ otro momento del ciclo de formación de las políticas públicas. Al mismo tiempo subraya la importancia de análisis diacrónicos que den seguimiento a los cambios ocurridos a lo largo del tiempo con una misma política. ¿Cómo es que el modelo de reforma adoptado modificó la configuración de actores, agendas y paradigmas involucrados previamente en la prestación de servicios de salud? ¿Qué es atribuible a intereses particulares y qué a los efectos del nuevo paradigma implementado? ¿Fueron estos actores los mismos o fueron otros? Las IFIs, ¿han tenido igual protagonismo? ¿Qué responsabilidad le cabe a la economía de salud como paradigma de gestión de los servicios de salud en la vulnerabilidad que la reforma conllevó para el despliegue de intereses particulares? Concretamente, se requiriere replicar el análisis para fases más avanzadas de la implementación de la política.

Simultáneamente, el estudio comparado de políticas pasadas y contemporáneas y de distintas etapas del ciclo de formación de las políticas, contribuiría a colocar el estudio de la realidad latinoamericana en la corriente principal del debate teórico y empírico internacional sobre la política pública.

\section{NOTAS}

${ }^{1}$ Es más frecuente encontrar estudios que ubican la política doméstica en el escenario internacional enfocados en momentos históricos previos. Tal es el caso del análisis de Rosenberg (1983) sobre la adopción de la seguridad social en Costa Rica a inicios de la década de los 40.

2 Por una sistematización de distintos enfoques y referentes empíricos aplicados a la política social, ver Martínez Franzoni, 2000.

3 Si bien existe el Ministerio de Salud, la Caja Costarricense del Seguro Social - CCSS monopoliza el financiamiento y la entrega de servicios de salud y, por lo tanto, es el principal objeto de las reformas institucionales (Mesa-Lago, 1994).

${ }^{4}$ El grueso del análisis fue previamente publicado en "Poder y alternativas: las agendas internacionales en las reformas del sector salud en Costa Rica, 1988-1998", Anuario Centroamericano de Estudios Sociales, v. 25, n. 1, 1999. La primera versión de este trabajo fue realizada para los efectos de ser presentada en la conferencia bianual de la Latin American Studies Association - LASA que tuvo lugar en Chicago en septiembre de 1998 adonde se recibieron valiosas observaciones e ideas por parte de Fernando Filgueira. Versiones posteriores se beneficiaron de agudos comentarios teóricos de Verónica Montecinos y observaciones empíricas de Juan Diego Trejos. Por supuesto, como siempre en estos casos, cualquier error de apreciación corre por cuenta de la autora del trabajo.

${ }^{5}$ Un ejemplo del paradigma de análisis de poder e intereses se encuentra en Esping-Andersen (1990). Un análisis que subraya el papel de las ideas y el aprendizaje se puede encontrar en Heclo (1974). La utilidad de combinar ambas perspectivas se puede encontrar en Martínez Franzoni (2000). En América Latina, el ascenso de los economistas y de sus ideas en la formación de políticas macroeconómicas ha sido abordado por Montecinos \& Markoff (1993) y por Markoff \& Montecinos (1993).

6 El PLN promovió la expansión y consolidación del estado social costarricense desde los años 50 . El estado ha sido el principal canal de movilidad ocupacional y social de los sectores medios, especialmente los profesionales, e históricamente ha representado a los grupos beneficiarios del modelo de sustitución de importaciones (González \& Céspedes, 1993; Garita, 1989). Por su parte, la coalición de partidos opositores, reunida en los 80 y 90 en el PUSC, históricamente ha representado a quienes se inclinan por acciones más decididamente de corte neoliberal en relación a asuntos tales como la apertura de economía, la participación de agentes privados y la reducción del papel del estado. Una descripción de las contiendas electorales desde 1950 se puede encontrar en Carey (1996). 
7 El PLN bajo Arias y el PUSC bajo Calderón hijo (cuyos gobiernos comenzaron en 1986 y 1990, respectivamente) alcanzaron la presidencia con apenas algo más de un $50 \%$ del total de los votos y tuvieron mayoría legislativa. Por el contrario, el PLN bajo Figueres Jr. y el PUSC bajo Rodríguez (1994 y 1998, respectivamente) ganaron las elecciones con menos del 50\% de los votos del país y no contaron con mayoría legislativa (Booth, 1998).

${ }^{8}$ La administración Arias dio continuidad a la administración Monge. Ambas administraciones llevaron adelante medidas heterodoxas que fueron gradualmente desmantelando el modelo de sustitución de importaciones desarrollado en Costa Rica a lo largo de los años 60 y 70 (Wilson, 1994).

${ }^{9}$ En particular, la administración Arias promovió la construcción masiva de viviendas para atender a poblaciones de inmigrantes del campo y de países vecinos como Nicaragua, precariamente asentadas en el cinturón de San José, capital del país.

10 En realidad, las cooperativas no fueron el primer intento reformista (antes se habían llevado a cabo los programas de médico de empresa y de libre elección médica). Sin embargo, sí constituyeron el primero con una proyección reformista de los arreglos institucionales del sector en su conjunto.

${ }^{11}$ Estas iniciativas permanecieron en papel, dado que estas conversaciones tuvieron lugar al mismo tiempo que emergía el camino alternativo de reforma

${ }^{12}$ Evidencia del desdibujamiento de las diferencias partidarias relativas a la reforma fue presentado en abundancia en una de las conversaciones personales con el Dr. Salas (1997), presidente ejecutivo de la CCSS durante el período 1994-1998. Sin embargo, esto no supuso desconocer amenazas a dicha reforma por parte de algunos sectores partidarios, especialmente del PUSC.

${ }^{13}$ El papel de las agencias internacionales en la ejecución de políticas de salud costarricenses ha sido escasamente examinado. Sin embargo, el papel que tuvieron estas organizaciones (particularmente la U. S. Agency for International Development - Usaid y la Organización Panamericana de la Salud) durante los años 70 ha sido examinado por Morgan (1993). Su análisis sugiere que dichas políticas fueron sencillamente impuestas a la población por estas organizaciones con la complicidad de las autoridades nacionales. Sin embargo, desde mi punto de vista, Morgan no presenta evidencia empírica que sostenga su argumento.

14 Antes de las reformas estructurales, tanto el Banco Mundial como el BID habían financiado programas sociales, predominantemente aspectos infraestructurales de dichos programas. Por ejemplo, bajo la presidencia de McNamara, el Banco Mundial había destinado recursos al financiamiento de proyectos de vivienda, saneamiento y desarrollo rural. También, durante los años 1970, el BID había financiado programas sociales, principalmente destinados a infraestructura, tales como saneamiento y desagües (Oliver, 1997). Una vez iniciado el ajuste estructural, sin embargo, no fue sino hasta comienzos de los años 90 que el Banco Mundial y las IFIs, en general, reconocieron la importancia, si bien instrumental, de combatir la pobreza y expandir la agenda social más allá de la agenda genérica de privatización y descentralización.

15 Este paquete sería el segundo Programa de Ajuste Estructural - PAE negociado desde 1988 y firmado en 1989. El primer PAE había sido acordado en 1985 y perseguía la reducción del papel del estado en la economía, el número de empleados del estado y el recorte del gasto público. Durante la primera mitad de los años 80, Costa Rica refinanció su deuda externa con prestadores gubernamentales del Club de París (1983 y 1985) y obtuvo préstamos destinados a estabilizar la economía por parte del Fondo Monetario Internacional - FMI. El segundo PAE dio continuidad a los objetivos del primero y agregó el aumento del impuesto a la propiedad, mejoramiento de la recaudación impositiva, eliminación de subsidios a algunas empresas públicas y promoción de exportaciones (Booth, 1998).

16 Tales como seguridad social, salud y educación.

17 En tanto red que financia proyectos de investigación destinada a apoyar políticas, la Red de Centros, dependiente de la Oficina del Economista en Jefe del Banco Interamericano de Desarrollo, es un buen ejemplo de la prioridad dada por las agencias a dicha elaboración.

18 A partir de estos programas se expandieron los servicios de atención primaria y Costa Rica se convirtió en un país ejemplar en materia de logros sanitarios. Entre dichos logros, cabe destacar el rápido y sin precedente descenso de la mortalidad infantil, la cual pasó de algo más de 60 por mil a poco más de 20 por mil entre el inicio y el fin de la década de los 70 (Rosero-Bixby, 1986).

${ }^{19}$ De hecho, la OIT tuvo un papel central en la propia creación del seguro social costarricense durante los años 40. En ese momento, la OIT promovió el foro más importante de discusión regional en relación a la seguridad social y sirvió además como vehículo de intercambio entre los especialistas de diferentes países. Fue a través de instancias promovidas por la OIT que técnicos de distintos países se conocieron e intercambiaron experiencias, a su vez precursoras de intercambios horizontales entre países. Así, técnicos costarricenses llegarían por ejemplo a Chile en los años 40 y a México en los años 60 (Rosenberg, 1983). 
${ }^{20}$ El desplazamiento de las agencias específicamente sectoriales en parte refleja la composición profesional de cada una: en las primeras se trata de especialistas de la salud, tales como nutricionistas, expertos en atención primaria o, más en general, administradores de la salud. En el caso de las IFIs se trata de economistas, a veces con experiencia en reformas institucionales previas en el sector salud. Dado su énfasis en aspectos institucionales, las reformas actuales dejan muy escaso margen a profesiones especializadas en el sector, dado que éstas no están en condiciones de realmente disputar el área de conocimiento propia de los expertos 'generalistas' que encuadran las reformas, estos últimos representados por los economistas de la salud.

${ }^{21}$ Por ejemplo, a estudios sobre calidad y costos de los servicios hospitalarios o el contenido de los servicios de atención primaria.

22 Más concretamente, se trataba del hospital de Alajuela y de un número importante de puestos de salud.

${ }^{23}$ El papel del BID, así como también el papel de expertos del Banco Mundial en la elaboración de un proyecto reformista, merece una mayor atención, tal como lo sugiere otra conversación personal mantenida con el Dr. Salas en 1999, la cual arrojó nueva luz al respecto. Dicha evidencia, sin embargo, lejos de contradecir el argumento presentado en este trabajo, lo refuerza.

${ }^{24}$ Coordinación que también se venía llevando adelante en educación a través del Programa de Mejoramiento Educativo - Promece, ejecutado en la década de los 90 en Costa Rica y, con nombres similares, en otros países de la región latinoamericana.

25 A medida que las reformas avanzan, también avanza el conocimiento de las IFIs acerca de la política y características institucionales de los países y sectores en cuestión, por lo tanto tal vez tendiendo a restringir la discrecionalidad doméstica.

${ }^{26}$ Flexibilidad en el cumplimiento de estos objetivos y seguimiento de recomendaciones, ocurre también en el caso de Uruguay.

${ }^{27}$ En relación a la reforma de pensiones en América Latina, Mesa-Lago provee un análisis exhaustivo acerca de la postura de las IFIs en las reformas de los sistemas de pensiones en América Latina (Mesa-Lago, 1994).

28 Por ejemplo, en la CCSS, tanto Miranda (administración del PLN, 1982-1990) como Jiménez (administración del PUSC, 1990-1994), habían previamente dirigido los hospitales México y de Niños, respectivamente.

${ }^{29}$ Filgueira y Moraes (1998) señalan que es precisamente por esta razón que la construcción de consenso en torno a las reformas constituye una condición sine qua non para que éstas sean exitosas en ambos países.

30 Documentos elaborados por la Dirección de Servicios Médicos de Salud de la Gerencia Médica. Por ejemplo, ver Zamora \& Sáenz (1995).

31 En efecto, el Dr. Marín, entonces gerente de la cooperativa de Pavas, no solo participó sino que tuvo un papel protagónico en la elaboración de todo un componente de la reforma relativo al primer nivel de atención.

32 Si pensamos en las medidas de atención primaria promovidas en el mundo en general y en Costa Rica en particular durante los años 70, esta preocupación estaba lejos de ser nueva. Sin embargo, en aquel momento se trataba de políticas 'para pobres', mientras que ahora tenían como objetivo transformar las redes universales de servicios del que hacían uso otros sectores sociales.

33 Estas clínicas fueron La Peregrina e Integral de Coronado en las cuales, de distintas maneras, se buscó combinar atención primaria con algún grado de medicina especializada. Al mismo tiempo, ambas clínicas promovieron participación comunitaria la cual, siendo parte de la tradición institucional del Ministerio de Salud, era prácticamente ajena a la del seguro social (Marín, 1990).

34 Por una descripción de la reforma chilena, ver Sojo (1998).

35 Tomo prestada la noción de 'imitación selectiva' que forma parte del análisis de globalización de Robertson (1994).

36 Tal extrema racionalidad instrumental se refleja en el caso de la dinastía Meiji en Japón (Westney, 1987). Entre 1880 y 1920, dichas elites identificaron modelos organizacionales exitosos para la producción de ciertos servicios, desde correos a fuerzas armadas, y los copiaron con el fin de obtener similares resultados en su propio país. Los criterios aplicados por las elites costarricenses, en caso para imitar selectivamente otras experiencias, se discuten en Martínez (1998).

${ }^{37}$ Esto se aplica no solo a la salud sino a las políticas sociales en general. En este sentido agendas y negociaciones, ideas y recursos de poder, se refuerzan o tensionan mutuamente. 


\section{REFERENCIAS BIBLIOGRÁFICAS}

Anderson, James

[1975] 1979

Ayala, Norma

1996

Barths, Mario León

1997

Birn, Anne-Emanuelle 2005

Booth, John

1998

Carey, John 1996

Cercone, James 1996

DiMaggio, Paul; Powell, Walter 1983

Durán, Fabio 1997

Durán, Fabio 1995

Esping-Andersen, Gosta 1990

Fiedler, John; Rigoli, Félix 1991

Filgueira, Fernando;

Moraes, Juan Andrés 1998

Garita, Luis 1989

González Vega, Claudio; Céspedes, Víctor Hugo 1993

Haggard, Stephen 1986

Hall, Peter 1993

Hall, Peter 1986
Public policy making.

2. ed. New York: Holt, Rinehart \& Winston.

[Coordinadora del componente de modernización del modelo de servicios de atención de salud, Unidad de Modernización de la CCSS.] Conversación personal. San José, Costa Rica.

[Director de la Dirección de Servicios Técnicos de Atención de la Salud.] Conversación personal. San José, Costa Rica.

Buscando desesperadamente la descentralización: las políticas de salud mexicanas en dos épocas de reforma (los años 20 y 30 y la década de los 80). Dynamis, v. 25, p. 279-311.

Costa Rica: quest for democracy.

Boulder (CO): Westview Press.

Term limits and legislative representation.

Cambridge: Cambridge University Press.

[Coordinador del componente del modelo de asignación de recursos, Unidad Técnica de Modernización de la CCSS; miembro de la misión del Banco Mundial durante la negociación del préstamo destinado a la reforma de la CCSS.] Conversación personal. San José, Costa Rica.

The iron cage revisited: institutional isomorphism and collective rationality in organizational fields.

American sociological review, v. 48, p. 147-60.

[Director División Actuarial, CCSS.] Conversación personal. San José, Costa Rica.

[Director División Actuarial, CCSS.] Conversación personal. San José, Costa Rica.

The three worlds of welfare capitalism.

Cambridge: Polity Press; Princeton: Princeton University Press.

The Costa Rican social security funds alternative models: a case study of the cooperative-based, Coopesalud Pavas Clinic.

Washington (DC): U. S. Agency for International Development - Usaid.

Political environments, sector specific configurations and strategic devices: understanding institutional reform in Uruguay. Trabajo para el proyecto: The political economy of institutional reform in Latin America. Washington (DC): Inter-American Development Bank.

The bureaucratization of the Costa Rican state.

In: Edelman, Marc; Kenen, Joanne (ed.) The Costa Rica Reader. New York: Groove Weidenfeld, p. 137-40.

Costa Rica.

In: Rottenberg, Simon (ed.) A World Bank comparative study. London: Oxford University Press, p. 15-183.

The politics of adjustment: lessons from the IMF's extended fund facilities. In: Kahler, Miles (ed.) The politics of international debt. Ithaca (NY): Cornell University Press.

Policy paradigms, social learning, and the state: the case of economic policy-making in Britain. Comparative politics, v. 25, n. 3, p. 275-96.

Governing the economy: the politics of state intervention in Britain and France. Oxford, New York: Oxford University Press. 
Heclo, Hugh

1974

Ikenberry, John 1990

Jacobsen, John Kurt 1995

Jones, Charles [1970] 1984

Kahler, Miles 1989

Lowi, Theodore 1964

Marín, Fernando 1990

Markoff, John;

Montecinos, Verónica 1993

Martínez, Juliana 2000

Martínez, Juliana 1998

Mesa-Lago, Carmelo 1994

Miranda, Guido 1994

Miranda, Guido [1988] 1993

Montecinos, Verónica; Markoff, John 1993

Morgan, Lynn 1993

Morsley, Paul 1987

Oliver, Robert 1997

Palmer, Steven 1998

Raventós, Ciska 1996
Modern social policies in Britain $\mathcal{E}$ Sweeden: from relief to income maintenance. New Haven: Yale University Press.

The international spread of privatization policies: inducements, learning and policy bandwagoning. In: The political economy of public sector reform and privatization. Boulder (CO): Westview. p. 88-110.

Much ado about ideas: the cognitive factor in economic policy. World politics, v. 47, p. 283-310.

An introduction to the study of public policy. 3. ed. Belmont (CA): Brooks/Cole.

International financial institutions and the politics of adjustment. In: Nelson, Joan (ed.) The politics of economic adjustment. New Brunswick: Transaction Books.

American business, public policy case studies and political theory. World politics, v. xvi, july, p. 677-715.

Administración descentralizada de los servicios de salud por parte de cooperativas: el caso de Costa Rica.

Revista centroamericana de administración pública, v. 18, p. 37-49.

The ubiquitous rise of economists.

Journal of public policies, v. 13, n. 1, p. 37-68.

Luces y sombras en la formación y transformación de las políticas sociales en América Latina. (Cuaderno de Flacso, n. 117). San José, Costa Rica.

Policy environments and selective emulation in the making of health policies: the case of Costa Rica, 1920-1997.

Tesis de doctorado, University of Pittsburgh, Pittsburgh.

Changing social security in Latin America and the Caribbean: towards the alleviation of social costs of economic reform.

Boulder (CO); London: Lynne Rienner.

[Presidente ejecutivo de la CCSS, 1982-1990.] Conversación personal. San José, Costa Rica.

El seguro social en Costa Rica.

San José: Cendeisss.

Democrats and technocrats: professional economists and regime transitions in Latin America. Comparative journal of development studies, v. XIV, n. 1, p. 7-22.

Community participation in health.

New York (NY): Cambridge University Press.

Conditionality as bargaining process: structural adjustment lending, 1980-1986. Princeton: Princeton University Press.

George Woods and the World Bank.

Boulder (CO): Lynne Rienner.

Central American encounters with Rockefeller Public Health, 1914-1921. In: LeGrand, Catherine; Gilbert Joseph, Ricardo Salvatore (ed.) Close encounters of empire: writing the cultural history of U. S. - Latin American relations. Durham and London: Duke University Press, p. 311-32.

Instituciones políticas y democracia en la aprobación de las políticas de ajuste en Costa Rica. Tercer Congreso Centroamericano de Historia. San José, julio 16-18. 
Robertson, Roland 1994

Rose, Richard 1991

Rose, Richard 1984

Rosenberg, Mark 1983

Rosero-Bixby, Luis 1986

Sáenz, Luis 1996

Salas, Álvaro 1999

Salas, Álvaro 1996

Salas, Álvaro 1994

Sojo, Ana 1998

Thun, Mala 2003

Trejos, Juan Diego;

Güendell, Ludwig 1994

Vargas, Mauricio 1996

Vargas, Mauricio 1994

Westney, Eleanor 1987

Weyland, Kurt 2005

Wilson, Bruce 1998

Wilson, Bruce 1994

World Bank 1998

World Bank 1993

Zamora, Carlos; Sáenz, Luis Bernardo 1995
Theory, specificity and change: emulation, selective incorporation and modernization. American Sociological Association.

Lesson-drawing across nations.

Journal of public policy, v. 11, n. 1, p. 1-2.

Do parties make a difference?

New Jersey: Chathan House Publishers.

Las luchas por el seguro social.

San José: Ed. San José.

Infant mortality in Costa Rica: explaining the recent decline.

Studies in family planning, v. 17, n. 2, p. 57-65.

[Coordinador Unidad Técnica de Modernización de la CCSS.] Entrevista. San José, Costa Rica.

[Presidente ejecutivo de la CCSS.] Conversación personal.

San José, Costa Rica.

[Presidente ejecutivo de la CCSS.] Conversación personal. San José, Costa Rica.

[Presidente ejecutivo de la CCSS.] Conversación personal. San José, Costa Rica.

Reforma de la salud en Chile.

Borrador.

Sex, divorce and the family under Latin American dictatorships and democracies. Cambridge: Cambridge University Press.

Reformas recientes en sector salud de Costa Rica. Reformas de politicas, v. 18. Santiago de Chile: Cepal.

[Gerente de Coopesain.] Conversación personal. San José, Costa Rica.

[Gerente de Coopesain.] Conversación personal. San José, Costa Rica.

Imitation and innovation: the transfer of western organizational patterns to Meiji Japan. Cambridge (MA): Harvard University Press.

Theories of policy diffusion: lessons from Latin American pension reform. World politics, v. 57, n. 2, January 2005, p. 262-95.

Costa Rica: politics, economics and democracy. Boulder (CO); London: Lynne Rienner Publishers.

When social democrats choose neoliberal economic policies? Comparative politics, v. 26, n. 2, p. 149-68.

Beyond the Washington Consensus: institutions matter. Washington (DC).

Investing in health: world development report.

Washington (DC).

Variaciones en los servicios de salud bajo diferentes modalidades de atención. San José: CCSS.

Recibido para publicación en marzo de 2006.

Aprobado para publicación en mayo de 2006. 